\title{
Validación de un instrumento para el tamizaje de casos de diabetes tipo 2 y la vigilancia de personas en riesgo en México
}

\author{
Fernando Guerrero-Romero ${ }^{1}$ y Martha Rodríguez-Morán ${ }^{1}$
}

Forma de citar Guerrero-Romero F, Rodríguez-Morán M. Validación de un instrumento para el tamizaje de casos de diabetes tipo 2 y la vigilancia de personas en riesgo en México. Rev Panam Salud Publica. 2010;27(3): $181-6$.

RESUMEN Objetivo. Validar un método para el tamizaje de casos de diabetes tipo 2 y la vigilancia de personas en riesgo en una población del norte de México.

Métodos. El instrumento para el tamizaje de la diabetes tipo 2 (ITD) se desarrolló a partir de un análisis de regresión logística múltiple que permitió determinar la asociación entre el diagnóstico nuevo de diabetes (variable dependiente) y 11 factores de riesgo ya conocidos. Se realizaron validaciones interna (por el método de validación cruzada de conjuntos) y externa mediante el seguimiento de una cohorte de personas sanas. Para estimar el riesgo relativo (RR) de desarrollar diabetes tipo 2 se toma la puntuación total del ITD según los factores de riesgo de una persona y se lleva a una curva que ofrece la probabilidad de que esa persona desarrolle la enfermedad.

Resultados. De las 525 personas de la cohorte, 438 (83,4\%) completaron en promedio 7 años de seguimiento (entre 4,5 y 10 años), para un total de 2696 personas-año; 62 (14,2\%) personas desarrollaron diabetes durante el seguimiento. Las personas que por sus factores de riesgo acumularon 55 puntos presentaron un incremento significativo en el riesgo de desarrollar diabetes en 7 años $(R R=6,1$; IC95\%: 1,7 a 11,1), mayor aún cuando la puntuación era de 75 puntos $(R R=9,4$; IC95\%: 2,1 a 11,5).

Conclusiones. El ITD es una alternativa válida y de fácil utilización para el tamizaje de la diabetes tipo 2. Su aplicación permitirá extender los beneficios de las acciones de prevención de la enfermedad y el diagnóstico temprano, sin aumentar considerablemente los costos y con un uso minimo de los recursos de laboratorio.

Palabras clave Diabetes mellitus tipo 2; factores de riesgo; tamizaje masivo; vigilancia; México.

Las enfermedades crónico-degenerativas son responsables de $70 \%$ de las muertes en personas mayores de 30 años en todo el mundo (1), por lo que su control cons-

Instituto Mexicano del Seguro Social, Durango, México. La correspondencia se debe dirigir a Martha Rodríguez-Morán, Instituto Mexicano del Seguro Social, Siqueiros No. 225, Zona Centro, Durango 34000, México. Correo electrónico: rodriguez moran@hotmail.com tituye uno de los principales retos de salud pública para el siglo XXI (2). Sin embargo, como tanto las enfermedades crónico-degenerativas como sus factores de riesgo son clínicamente silentes en las etapas iniciales de su evolución, para llegar a un diagnóstico oportuno se requieren acciones específicas dirigidas a su detección (2). La prevalencia de diabetes tipo 2, una de estas enfermedades, ha mostrado un rápido incremento en los últimos años (3), por lo que reducir su incidencia es una prioridad de las políticas de salud pública en todos los países, tanto desarrollados como en vías de desarrollo (4).

El reconocimiento temprano y la atención preventiva oportuna de las personas en riesgo de padecer diabetes tipo 2 podrían ayudar a reducir la incidencia de esta enfermedad $(5,6)$, por lo que se han propuesto diversas estrategias de ta- 
mizaje dirigidas a la población en general y a los grupos de riesgo. Sin embargo, las acciones de tamizaje, aunque benéficas, tienen costos elevados (7) y muchos autores consideran que aún no se cuenta con datos científicos suficientemente sólidos que permitan recomendar la generalización de su aplicación $(8,9)$.

La principal controversia relacionada con las pruebas de tamizaje se centra en la falta de estudios que demuestren los beneficios de las acciones de prevención de la diabetes (10). En una intervención diseñada para reducir mediante medidas no farmacológicas el riesgo de desarrollar diabetes y que contó con un año de seguimiento, Gillies y colaboradores (11) estimaron que el costo del tamizaje era aproximadamente de US\$ 9,85 por persona y la probabilidad de que la intervención fuera rentable en términos de años con calidad de vida ganados era de $93,0 \%$. Sin embargo, otros autores afirman que la detección de la diabetes mediante la medición de la glucemia en ayunas no es efectiva (12). En este contexto, se debe mencionar que de 347 personas en las que se detectaron valores elevados de glucosa en sangre capilar durante un programa de tamizaje dirigido a la población general, solo 16 $(4,6 \%)$ acudieron a realizarse la prueba confirmatoria y el diagnóstico de prediabetes o diabetes se logró establecer en 14 de ellas (4,0\%); el costo directo de ese programa de tamizaje fue de US\$ 776,00 por caso detectado (13). La baja tasa de respuesta de las personas en riesgo para someterse al estudio diagnóstico confirmatorio puso de relieve que el ambiente sociocultural y el nivel educacional de la población pueden influir en los resultados de los programas de tamizaje (13).

Por otro lado, se han propuesto diversos cuestionarios para identificar a personas que podrían estar en riesgo de desarrollar diabetes o intolerancia a la glucosa, lo que podría ayudar a reducir los costos del tamizaje (14-16). Sin embargo, esos instrumentos se han desarrollado para poblaciones anglosajonas $\mathrm{y}$, aún cuando los factores de riesgo para el desarrollo de la diabetes no varían significativamente de una población a otra, las diferentes condiciones socioculturales y el disímil nivel educacional impiden su utilización en otras poblaciones sin una previa adaptación y validación.

Por ello, el objetivo de este estudio fue validar un método para el tamizaje de casos de diabetes tipo 2 y la vigilancia de personas en riesgo en una población del norte de México.

\section{MATERIALES Y MÉTODOS}

El instrumento para el tamizaje de la diabetes tipo 2 (ITD) se desarrolló a partir de un análisis de regresión logística múltiple que permitió determinar la asociación entre un diagnóstico nuevo de diabetes (variable dependiente) y 11 factores de riesgo ya conocidos (variables independientes): sexo; edad; anteceden- tes familiares de diabetes, hipertensión y obesidad; antecedentes de diabetes gestacional o macrosomía; sedentarismo; hipertensión arterial; índice de masa corporal (IMC); y niveles de trigliceridemia y glucemia en ayunas. Mediante este análisis, basado en la información obtenida de 711 personas, se calculó el valor del coeficiente beta, que expresa la asociación entre cada variable independiente y la presencia de diabetes. El valor del coeficiente beta de cada variable independiente se transformó a su valor porcentual, de forma que todos los coeficientes obtenidos sumaran $100 \%$ (figura 1).

El instrumento se validó interna y externamente. Para lo primero se utilizó el método de validación cruzada de conjuntos (v-fold cross validation) (17), en el que cada variable (modelo de formación) se contrastó contra la variable dependiente en un modelo ajustado por las variables restantes y esto se repitió para todos los modelos de formación. Las pruebas de todos los modelos de formación fueron similares a la original.

Para la validación externa se integró una cohorte de personas sanas mediante un procedimiento aleatorio en dos etapas; en la primera se eligieron de manera aleatoria 20 áreas geográficas estadísticas básicas (AGEB) de la ciudad de Durango, capital del Estado de Durango, México, representativas de los diferentes estratos socioeconómicos y culturales. En la segunda se eligió de manera aleatoria un número igual de manzanas de

\section{FIGURA 1. Valores porcentuales de los coeficientes beta de los factores de riesgo y curva para calcular el riesgo de diabetes ${ }^{\mathrm{a}}$ en siete años}

\begin{tabular}{|l|c|}
\hline \multicolumn{1}{|c|}{ Variable } & $\begin{array}{c}\text { Puntos } \\
(\%)\end{array}$ \\
\hline Mujer & 4,9 \\
\hline Edad $\geq 40$ años & 12,3 \\
\hline Antecedentes familiares de diabetes tipo 2 & 10,1 \\
\hline Antecedentes familiares de hipertensión arterial & 4,8 \\
\hline Antecedentes familiares de obesidad & 4,2 \\
\hline $\begin{array}{l}\text { Antecedentes de diabetes gestacional } \\
\text { o macrosomía }\end{array}$ & 5,7 \\
\hline Sedentarismo & 5,5 \\
\hline Presión sistólica $\geq 135 \mathrm{mmHg}$ o diastólica $\geq 85 \mathrm{mmHg}$ & 6,9 \\
\hline Obesidad, índice de masa corporal $\geq 30$ & 13,9 \\
\hline Glucosa en ayunas $\geq 5,6 \mathrm{mmol} / \mathrm{L}$ (100 $\mathrm{mg} / \mathrm{dL})$ & 15,3 \\
\hline Triglicéridos $\geq 1,7 \mathrm{mmol} / \mathrm{L}$ (150 $\mathrm{mg} / \mathrm{dL})$ & 16,4 \\
\hline
\end{tabular}

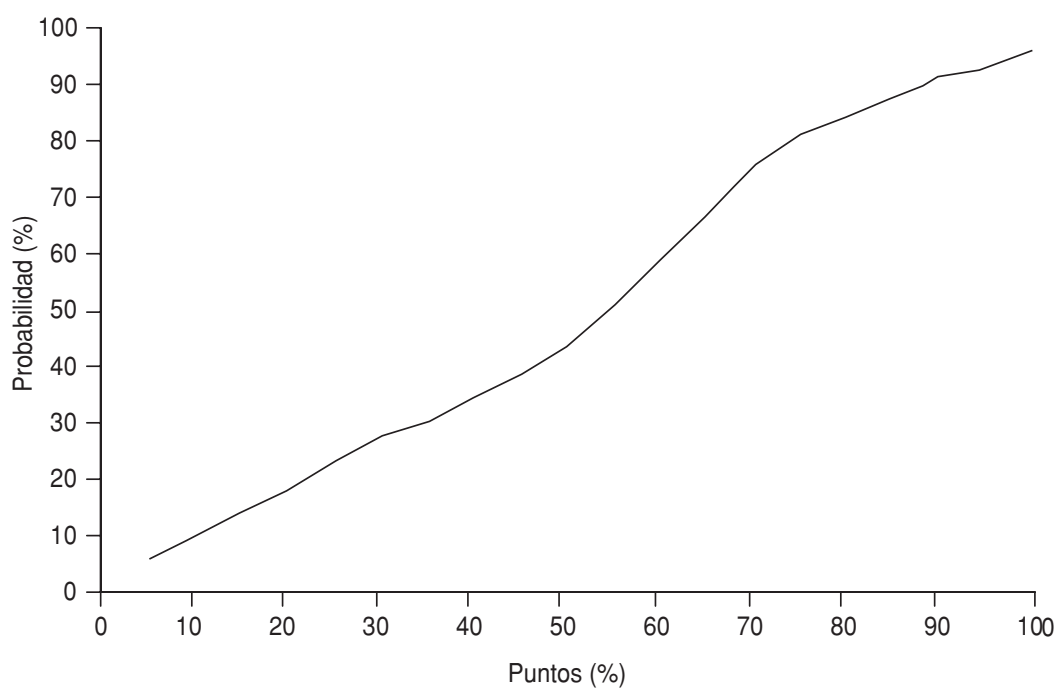

a Para estimar el riesgo de diabetes: a) se ubica en el eje de las abscisas el valor de la suma de los factores de riesgo de una persona según el cuadro; b) desde este punto se traza una línea perpendicular al eje de las abscisas hasta la curva; c) desde el punto de intersección con la curva se traza una línea paralela al eje de las abscisas hasta el eje de las ordenadas, que indicará la probabilidad de desarrollar la enfermedad. 
cada AGEB. Investigadores de campo visitaron todos los domicilios de las manzanas seleccionadas para invitar a las personas elegibles a participar en el estudio. La cohorte, integrada a partir de febrero de 1996, quedó conformada por 525 adultos sanos de 20 a 65 años de edad, los que recibirían seguimiento durante varios años para detectar el posible desarrollo de diabetes tipo 2. Se excluyeron las personas con diagnóstico previo de diabetes tipo 2 o que recibieron ese diagnóstico durante la medición inicial realizada para integrar la cohorte de estudio. Se informó de su condición de riesgo a las personas obesas o que presentaron valores elevados de glucemia en ayunas o intolerancia a la glucosa, y se les proporcionaron consejos nutricionales (18) y sobre la realización de ejercicios físicos.

A partir del valor del ITD al inicio y al final de la investigación, así como del desenlace (diagnóstico de diabetes tipo 2 o no) de cada persona de la cohorte se elaboró una curva para determinar la probabilidad de que una persona desarrolle la enfermedad (figura 1).

Se estimaron los valores de sensibilidad, especificidad, valor predictivo positivo, valor predictivo negativo y el área bajo la curva ROC (receiver operating characteristic) del ITD (19). Finalmente, y teniendo en consideración solo las variables clínicas, se estableció el punto de corte - es decir, el valor que ofreció mayores cifras de sensibilidad y especificidad - para detectar las personas con probabilidad real de desarrollar diabetes tipo 2.

\section{Definiciones}

Se consideró que una persona tenía antecedentes familiares de diabetes, hipertensión u obesidad si al menos un familiar de primer grado había presentado alguno de esos trastornos. El sedentarismo se definió como la falta de ejercicio programado o cuando este se realizaba durante menos de 50 minutos a la semana. La diabetes gestacional se estableció si durante un embarazo previo, la mujer presentó dos mediciones de glucemia en ayunas iguales o mayores de $7 \mathrm{mmol} / \mathrm{L}$ (126 mg/dL) después de la semana 24 de gestación, un valor de glucemia en ayunas mayor de $11,1 \mathrm{mmol} / \mathrm{L}$ (200 mg/dL) o una glucemia poscarga oral de $50 \mathrm{~g}$ de glucosa mayor o igual de $8,6 \mathrm{mmol} / \mathrm{L}$ (155 mg/dL) (20). Se consi- deró que había antecedentes de macrosomía si el producto de un parto anterior había pesado al nacer $4 \mathrm{~kg}$ o más; sobrepeso, si el IMC $\geq 25$; obesidad, cuando el IMC $\geq 30$; hipertensión arterial, cuando las cifras de presión arterial sistólica o diastólica eran igual o mayores de 135 $\mathrm{mmHg}$ u $85 \mathrm{mmHg}$, respectivamente (21); valores elevados de glucemia, si la concentración de glucosa plasmática en ayuno de 8 horas $\geq 5,6 \mathrm{mmol} / \mathrm{L}$ (100 $\mathrm{mg} / \mathrm{dL}$ ) (22); e hipertrigliceridemia, si la concentración sérica de triglicéridos en ayuno de 12-14 horas $\geq 1,7 \mathrm{mmol} / \mathrm{L}$ (150 $\mathrm{mg} / \mathrm{dL}$ ) (23).

La diabetes se definió de acuerdo con los criterios establecidos por la Asociación Estadounidense de Diabetes (22).

\section{Procedimiento}

El tamizaje de diabetes con el ITD consiste en una evaluación en dos pasos. El primero abarca solamente el análisis de las variables clínicas, por lo que el máximo puntaje que se puede obtener es de 68,3 (figura 1); el segundo abarca mediciones de laboratorio que, para reducir los costos del tamizaje, se indicarían solamente a las personas que en la evaluación clínica acumularon 35 puntos o más, lo que corresponde aproximadamente a un riesgo de $30,0 \%$ de desarrollar diabetes.

Así, durante la vigilancia de las personas en riesgo, la disminución (o incremento) en el riesgo de desarrollar diabetes se cuantifica según la disminución (o incremento) del puntaje obtenido en el ITD en cada visita. Esto permite enfocar las actividades de intervención dirigidas a prevenir la enfermedad hacia las variables modificables que permanezcan sin cambio.

Para estimar la probabilidad de una persona de desarrollar diabetes se requiere establecer cuáles de los factores de riesgo incluidos en el ITD están presentes; la suma de los valores de esos factores se traslada al eje de las abscisas de la curva del ITD (figura 1). Desde este punto se traza una línea vertical hasta la curva y de este punto se traza una línea horizontal hasta el eje de las ordenadas, donde se encuentra el valor de la probabilidad (expresada en porcentaje) de desarrollar diabetes en 7 años (figura 1). Por ejemplo, una mujer de 45 años de edad, con obesidad, sedentarismo, antecedente de macrosomía y antecedentes familiares de diabetes (52,4 puntos en la evaluación clínica), que presenta además niveles de trigliceridemia y glucemia en ayunas de $2,4 \mathrm{mmol} / \mathrm{L}(210 \mathrm{mg} / \mathrm{dL})$ y $6,4 \mathrm{mmol} / \mathrm{L}$ (115 mg/dL), respectivamente, tendrá un total de 84,1 puntos, por lo que su probabilidad de desarrollar diabetes sería de $87,0 \%$. En el caso hipotético de que la persona realice ejercicio y reduzca su IMC por debajo de 25 y sus niveles de trigliceridemia y glucemia en ayunas desciendan a valores inferiores de 1,7 mmol/L (150 mg/dL) y 5,6 mmol/L (100 mg/dL), respectivamente, el puntaje disminuiría a 33 puntos y, por lo tanto, la probabilidad de desarrollar diabetes se reduciría a $29,0 \%$.

\section{Análisis estadístico}

Para el análisis de los datos de la cohorte de validación externa, los valores se expresaron como promedios con su desviación estándar. Las diferencias entre los grupos se estimaron con la prueba de la $t$ de Student para grupos independientes y la prueba de la U de Mann-Whitney para los datos sin distribución no normal; se utilizó la prueba de la ji al cuadrado para las diferencias entre proporciones.

Para estimar el riesgo relativo (RR) de desarrollar diabetes (variable dependiente) se utilizó un modelo de regresión de Poisson en el que la variable independiente fue el valor igual a 55 puntos, que se deriva de la suma de los factores de riesgo que corresponden aproximadamente a una probabilidad de $50,0 \%$ de desarrollar diabetes (figura 1). Se calcularon el número de eventos por cada 100 personas-año (tasa cruda de incidencia) y los intervalos de confianza de $95 \%$ (IC95\%). Se estableció un nivel de significación estadística de $P<0,05$. Los datos se procesaron con el programa estadístico SPSS versión 15.0.

Este estudio recibió la aprobación del Comité de Investigación y Ética del Instituto Mexicano del Seguro Social de la ciudad de Durango, Durango, México.

\section{RESULTADOS}

La cohorte empleada para la validación externa del ITD quedó integrada por 525 personas. De ese total, $438(83,4 \%)-307$ $(70,1 \%)$ mujeres y $131(29,9 \%)$ hombrescompletaron en promedio 7 años de seguimiento (entre 4,5 y 10 años), para un total de 2696 personas-año. No se pudo lograr el seguimiento de $87(16,6 \%)$ per- 
sonas por haber cambiado su domicilio afuera de la ciudad. Sesenta y dos personas $(14,2 \%)$ desarrollaron diabetes durante el seguimiento (tasa cruda de incidencia $=2,3$ eventos $/ 100$ personas-año).

$\mathrm{Al}$ inicio de la investigación, los valores promedio de presión arterial (sistólica y diastólica) y niveles de trigliceridemia eran significativamente más elevados en los hombres que en las mujeres, mientras que los niveles de glucemia en ayunas fueron más elevados en las mujeres (cuadro 1).

En comparación con las personas que no desarrollaron diabetes durante el seguimiento, las que desarrollaron la enfermedad tenían mayor edad al inicio de la investigación, eran más obesas, tenían mayor presión arterial sistólica y sus niveles de trigliceridemia y glucemia en ayunas eran más elevados (cuadro 2). De igual manera, los factores de riesgo considerados en el ITD fueron significativamente más frecuentes en las personas que desarrollaron diabetes que en las que no la desarrollaron, con excepción del sexo (figura 2).

En general, $55(88,7 \%)$ de las 62 personas que desarrollaron diabetes presentaron al inicio del seguimiento puntuaciones superiores al valor de corte establecido de 35 puntos. Se obtuvieron altos valores de sensibilidad $(92,0 \%)$, especificidad $(71,0 \%)$, valor predictivo positivo $(35,0 \%)$, valor predictivo negativo $(97,5 \%)$ y área bajo la curva ROC $(0,91)$.

Las personas que por sus factores de riesgo acumularon 55 puntos presentaron un incremento significativo en el riesgo de desarrollar diabetes en 7 años (RR = 6,1; IC95\%: 1,7 a 11,1), mayor aún cuando la puntuación era de 75 puntos $(R R=9,4$; IC95\%: 2,1 a 11,5). Sin embargo, no se observó incremento alguno en el riesgo de desarrollar la enfermedad cuando la puntuación era de 35 puntos o menos (RR = 2,1; IC95\%: 0,9 a 10,7).

\section{DISCUSIÓN}

En general, se recomienda realizar el tamizaje de enfermedades que representan un problema de salud importante, su historia natural es conocida, su diagnóstico en etapas tempranas es posible y el tratamiento oportuno puede mejorar el pronóstico de la enfermedad (5). Estas condiciones se cumplen en muchas enfermedades crónicas, en particular, en la diabetes tipo 2. Por ello, el tamizaje dirigido a personas asintomáticas a partir de la detección de factores de riesgo asocia- dos con el desarrollo de la diabetes tipo 2 es un tema de salud pública importante y vigente, ya que permitiría aplicar acciones de prevención primaria.

El Comité de Expertos de la Asociación Estadounidense de Diabetes (24) recomienda el tamizaje de personas aparentemente sanas mayores de 45 años que tengan un IMC $\geq 25$ y de personas de cualquier edad con IMC $\geq 25$ que presenten además uno o más de los siguientes factores de riesgo: antecedentes familiares de diabetes, antecedentes de enfermedad vascular, sedentarismo, niveles elevados de glucemia en ayunas, intolerancia a la glucosa, niveles bajos de colesterol por lipoproteínas de alta densidad (HDL), hipertrigliceridemia, ovarios poliquísticos, antecedentes de diabetes gestacional o macrosomía.

El ITD se basa en una escala cuantitativa y sus costos son muy bajos, ya que las pruebas de laboratorio se indican solo a las personas con más de 35 puntos en la evaluación clínica inicial. Esto hace que se pueda extender el tamizaje a una población más amplia, independientemente de la edad o la ausencia de obesidad. Esta es la principal ventaja del ITD, ya que extiende los beneficios del tamizaje a las personas que a pesar de tener

CUADRO 1. Características iniciales de la cohorte de validación externa ${ }^{\mathrm{a}}$

\begin{tabular}{|c|c|c|c|c|}
\hline Variable & $\begin{array}{c}\text { Total } \\
n=438\end{array}$ & $\begin{array}{c}\text { Hombres } \\
n=131\end{array}$ & $\begin{array}{c}\text { Mujeres } \\
n=307\end{array}$ & $P^{b}$ \\
\hline Edad (años) & $42,5 \pm 9,7$ & $42,5 \pm 14,1$ & $42,5 \pm 11,4$ & 0,99 \\
\hline Presión arterial sistólica (mmHg) & $116,9 \pm 26,7$ & $123,1 \pm 23,4$ & $114,2 \pm 17,9$ & 0,0001 \\
\hline Presión arterial diastólica (mmHg) & $73,8 \pm 16,8$ & $76,9 \pm 10,4$ & $72,5 \pm 10,9$ & $<0,0001$ \\
\hline Índice de masa corporal & $29,4 \pm 6,7$ & $29,0 \pm 4,4$ & $29,5 \pm 5,5$ & 0,31 \\
\hline Glucemia en ayunas (mmol/L) & $5,6 \pm 0,5$ & $5,4 \pm 0,4$ & $5,6 \pm 0,4$ & $<0,0001$ \\
\hline Trigliceridemia (mmol/L) & $2,3 \pm 2,0$ & $2,6 \pm 2,7$ & $2,1 \pm 1,5$ & 0,04 \\
\hline
\end{tabular}

CUADRO 2. Características de la cohorte de validación externa al inicio y al final del período de seguimiento

\begin{tabular}{|c|c|c|c|c|c|c|}
\hline \multirow[b]{2}{*}{ Variable } & \multicolumn{3}{|c|}{ Al inicio del seguimiento } & \multicolumn{3}{|c|}{ Al final del seguimiento } \\
\hline & $\begin{array}{c}\text { Desarrollaron } \\
\text { diabetes } \\
(n=62)\end{array}$ & $\begin{array}{c}\text { No } \\
\text { desarrollaron } \\
\text { diabetes } \\
(n=376)\end{array}$ & $P^{a}$ & $\begin{array}{l}\text { Desarrollaron } \\
\text { diabetes } \\
(n=62)\end{array}$ & $\begin{array}{c}\text { No } \\
\text { desarrollaron } \\
\text { diabetes } \\
(n=376)\end{array}$ & $P^{a}$ \\
\hline Edad (años) & $51,4 \pm 10,0$ & $41,0 \pm 11,9$ & $<0,0001$ & $58,5 \pm 8,5$ & $47,9 \pm 8,4$ & $<0,0001$ \\
\hline Presión arterial sistólica $(\mathrm{mmHg})$ & $125,4 \pm 20,4$ & $115,4 \pm 19,7$ & 0,0003 & $145,1 \pm 15,1$ & $121,8 \pm 13,7$ & $<0,0001$ \\
\hline Presión arterial diastólica $(\mathrm{mmHg})$ & $76,0 \pm 10,9$ & $73,4 \pm 10,9$ & 0,08 & $85,1 \pm 9,1$ & $74,5 \pm 8,4$ & $<0,0001$ \\
\hline Índice de masa corporal & $31,0 \pm 3,9$ & $29,2 \pm 5,3$ & 0,001 & $32,1 \pm 2,7$ & $29,4 \pm 4,8$ & $<0,0001$ \\
\hline Glucemia en ayunas (mmol/L) & $6,7 \pm 0,4$ & $5,4 \pm 0,6$ & $<0,0001$ & $8,1 \pm 0,8$ & $5,5 \pm 1.0$ & $<0,0001$ \\
\hline Trigliceridemia (mmol/L) & $4,8 \pm 4,0$ & $1,9 \pm 1,0$ & $<0,0001$ & $4,9 \pm 2,7$ & $2,1 \pm 0,9$ & $<0,0001$ \\
\hline
\end{tabular}

a Según la prueba de la $t$ de Student para muestras independientes, excepto para la trigliceridemia en que se utilizó la prueba de la U de Mann Whitney. Nivel de significación $P<0,05$. 
FIGURA 2. Factores de riesgo a al inicio de la investigación en las personas de la cohorte de evaluación

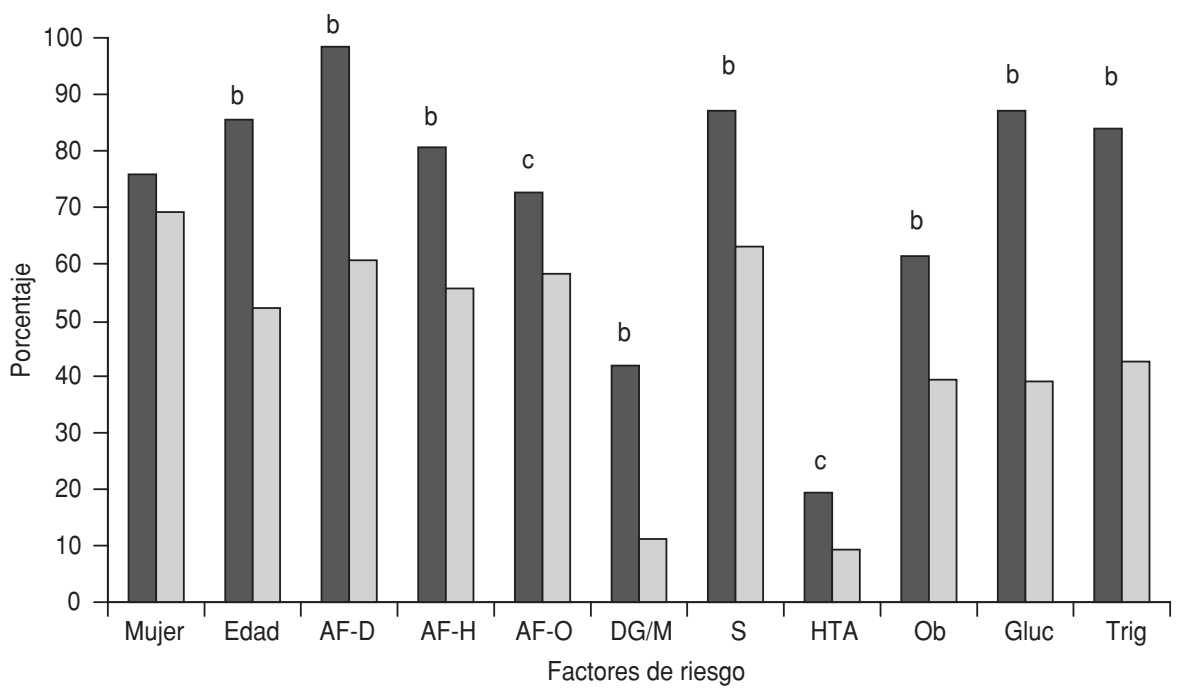

Personas que desarrollaron diabetes $\quad \square$ Personas que se mantuvieron sin diabetes

a Edad: $\geq 40$ años; AF-D: antecedentes familiares de diabetes; AF-H: antecedentes familiares de hipertensión arterial; AF-O antecedentes familiares de obesidad; DG/M: antecedentes de diabetes gestacional o macrosomía; S: sedentarismo; HTA: hipertensión arterial (presión arterial sistólica $\geq 135 \mathrm{mmHg}$ o diastólica $\geq 85 \mathrm{mmHg}$ ); Ob: obesidad (índice de masa corporal $\geq 30$ ); Gluc: glucemia en ayunas $\geq 5,6 \mathrm{mmol} / \mathrm{L}$ (100 mg/dL); Trig: trigliceridemia $\geq 1,7 \mathrm{mmol} / \mathrm{L}$ (150 mg/dL).

b Diferencia significativa $(P<0,0001)$

c Diferencia significativa $(P<0,05)$.

un peso normal presentan alteraciones metabólicas características de las personas obesas (25) y que constituyen un grupo de alto riesgo de diabetes y enfermedad cardiovascular. Además, el ITD es también útil para evaluar de forma cuantitativa el progreso de las intervenciones de prevención primaria en las personas de grupos de riesgo.

Recientemente, en un estudio de seguimiento, Kahn y colaboradores (26) derivaron y validaron un método de predicción del desarrollo de la diabetes tipo 2 con un sistema de puntuación que incluía los niveles de glucemia en ayunas, la circunferencia de la cintura, los antecedentes familiares de diabetes, la raza, la estatura, la edad, la hipertensión arte- rial, la taquicardia, el consumo de alcohol y los niveles séricos de triglicéridos, colesterol de HDL y ácido úrico. Ese instrumento presentó una sensibilidad de $74,0 \%$ (frente a $92,0 \%$ del ITD), especificidad de 71,0\% (igual al ITD) y ROC de 0,79 (frente a 0,91 del ITD). Otras recomendaciones para el escrutinio de la diabetes tipo 2 se basan en grupos de consenso $(8,25)$.

Además de las ventajas enumeradas, el ITD es el primer modelo predictivo de diabetes validado en una población latinoamericana. Su empleo en el tamizaje de la diabetes tipo 2 facilitará encausar las intervenciones dirigidas a reducir los factores de riesgo y mejorar el control de la hiperglucemia, con lo que se podría re- tardar la aparición de la diabetes tipo 2 y disminuir su prevalencia. No obstante, se requieren más estudios de seguimiento y evaluar la relación costo-beneficio de la aplicación de este instrumento, en términos de años de calidad de vida ganados.

Dado que el ITD se diseñó como una herramienta de tamizaje de bajo costo y que requiere poco tiempo para su aplicación, se complementa con pocas pruebas de laboratorio. Esto debe facilitar su utilización en los diferentes ámbitos de atención sanitaria en América Latina, incluidos los de mayores carencias y en los que no hay acceso a otras pruebas de laboratorio. El hecho de que la sensibilidad del instrumento con los datos de glucemia en ayunas fuera de $97,5 \%$ hace que la adición de otras mediciones (como la glucemia poscarga) eleven su sensibilidad solamente de forma marginal. No obstante, se debe tener presente que el ITD se validó en una población del norte de México cuyas características socioculturales, económicas, nutricionales y genéticas pueden ser específicas, por lo que se deberá validar en otras poblaciones latinoamericanas.

El ITD resultó una alternativa válida y de fácil utilización para el tamizaje de la diabetes tipo 2. Su aplicación permitirá extender los beneficios de las acciones de prevención de la enfermedad y el diagnóstico temprano, sin aumentar considerablemente los costos y con un uso mínimo de los recursos de laboratorio.

Agradecimientos. Se agradece al personal de la Unidad de Investigación Biomédica del Instituto Mexicano del Seguro Social (IMSS), en Durango, que participó entusiastamente en el desarrollo del proyecto. Este trabajo recibió financiamiento parcial de la Fundación IMSS, A.C., de México.

\section{REFERENCIAS}

1. Wild S, Roglic G, Sicree R, Green A, King H. Global burden of diabetes mellitus in the year 2000. Global burden of disease. Geneva: World Health Organization; 2003.

2. Nathan DM, Herman WH. Screening for diabetes: can we afford not to screen? Ann Intern Med. 2004;140(9):756-8.

3. Wild S, Roglic G, Green A, Sicree R, King H. Global prevalence of diabetes. Estimates for the year 2000 and projections for 2030. Diabetes Care. 2004;27(5):1047-53.
4. Thompson WG. Early recognition and treatment of glucose abnormalities to prevent type 2 diabetes mellitus and coronary heart disease. Mayo Clin Proc. 2001;76(11):1137-43.

5. The CDC Diabetes Cost-Effectiveness Study Group. The cost-effectiveness of screening for type 2 diabetes. J Am Med Assoc. 1998; 280(20):1757-63.

6. Edelman D, Olsen MK, Dudley TK, Harris AC, Oddone EZ. Impact of diabetes screening on quality of life. Diabetes Care. 2002;25(6):1022-6.
7. Hoerger TJ, Harris R, Hicks KA, Donahue K, Sorensen S, Engelgau M. Screening for type 2 diabetes mellitus: a cost-effectiveness analysis. Ann Intern Med. 2004(9);140:689-99.

8. U.S. Preventive Services Task Force. Screening for type 2 diabetes mellitus in adults: recommendations and rationale. Ann Intern Med. 2003;138(3):215-29.

9. Norris SL, Kansagara D, Bougatsos CH, Fu R. Screening adults for type 2 diabetes: a review of the evidence for the U.S. Preventive Ser- 
vices Task Force. Ann Intern Med. 2008; 148(11):855-68.

10. Engelgau MM, Narayan KMV, Herman WH. Screening for type 2 diabetes. Diabetes Care. 2002,23(9):1563-80.

11. Gillies CL, Lambert PC, Abrams KR, Sutton AJ, Cooper NJ, Hsu RT, et al. Different strategies for screening and prevention of type $2 \mathrm{di}-$ abetes in adults: cost effectiveness analysis. $\mathrm{Br}$ Med J. 2008;336(7654):1180-5.

12. Lawrence JM, Bennett $P$, Young A, Robinson AM. Screening for diabetes in general practice: cross sectional population study. Br Med J. 2001;323(7312):548-51.

13. Rodríguez-Morán M, Alvarado-Ruiz R, Morales-Franco G, Guerrero-Romero F. Combinación de un cuestionario autoaplicable con la determinación de glucosa capilar en el tamizaje de diabetes tipo 2. Salud Publica Mex. 2008;50(4):272

14. Heikes KE, Eddy DM, Arondekar B, Schlessinger L. Diabetes risk calculator. A simple tool for detecting undiagnosed diabetes and pre-diabetes. Diabetes Care. 2008; 31(5):1040-5.

15. Franciosi M, De Berardis G, Rossi MC, Sacco M, Belfiglio M, Pellegrini F, et al. Use of the diabetes risk score for opportunistic screening of undiagnosed diabetes and impaired glucose tolerance: the IGLOO (Impaired Glucose Tolerance and Long-Term Outcomes Observational) study. Diabetes Care. 2005;28(5):1187-94.

16. Ealovega MW, Tabaei BP, Brandle M, Burke $\mathrm{R}$, Herman WH. Opportunistic screening for diabetes in routine clinical practice. Diabetes Care. 2004;27(1):9-12.

17. Breiman L, Friedman J, Olshen RA, Stone CJ. Classification and regression trees. Boca Raton, FL: Chapman and Hall/CRC; 1998.

18. Eriksson J, Lindstrom J, Valle $T$, Aunola $S$, Hamalainen H, Llanne-Parikka P, et al. Prevention of type II diabetes in subjects with impaired glucose tolerance: the Diabetes Prevention Study (DPS) in Finland. Study design and 1-year interim report on the feasibility of the lifestyle intervention programme. Diabetologia. 1999;42(7):793-801.

19. Kramer MS. Clinical epidemiology and biostatistics. A primer for investigators and decision-makers. Berlin: Springer-Verlag; 1988:201-10.

20. American Diabetes Association. Diagnosis and classification of diabetes mellitus. Diabetes Care. 2005;28(Suppl 1):S37-42.

21. Chobanian AV, Bakris GL, Black HR, Cushman WC, Green LA, Izzo JL Jr, et al. The National High Blood Pressure Education Program Coordinating Committee. Seventh report of the Joint National Committee on Prevention, Detection, Evaluation, and Treatment of High Blood Pressure. Hypertension. 2003;42(6):1206-52.

22. The Expert Committee on the Diagnosis and Classification of Diabetes Mellitus. Report of the Expert Committee on the Diagnosis and Classification of Diabetes Mellitus. Diabetes Care. 2003;26(Suppl 1):S5-20.

23. Garg A, Simha V. Update on dyslipidemia. J Clin Endocrinol Metab. 2007;92(5):1581-9.

24. American Diabetes Association. Screening for diabetes. Diabetes Care. 2003;26(Suppl. 1): S21-4.

25. Ruderman N, Chisholm D, Pi-Sunyer $X$ Schneider $S$. The metabolically obese, normalweight individual revisited. Diabetes. 1998; 47(5):699-713.

26. Kahn HS, Cheng YJ, Thompson TJ, Imperatore G, Gregg EW. Two risk-scoring systems for predicting incident diabetes mellitus in U.S. adults age 45 to 64 years. Ann Intern Med. 2009;150(11):741-51.

Manuscrito recibido el 17 de agosto de 2009. Aceptado para publicación, tras revisión, el 30 de octubre de 2009.

ABSTRACT Objective. To validate a method for screening cases of type 2 diabetes and monitoring at-risk people in a community in northern Mexico.

\section{Validation of an instrument for screening cases of type 2 diabetes and monitoring at-risk individuals in Mexico}

Methods. The screening instrument for type 2 diabetes (ITD, for its Spanish acronym) was developed using a multiple logistic regression analysis that made it possible to determine the association between a new diagnosis of diabetes (a dependent variable) and 11 known risk factors. Internal validations were performed (through v-fold cross-validation), together with external validations (through the monitoring of a cohort of healthy individuals). In order to estimate the relative risk (RR) of developing type 2 diabetes, the total ITD score is calculated on the basis of an individual's risk factors and compared against a curve that shows the probability of that individual developing the disease.

Results. Of the 525 people in the cohort, 438 (83.4\%) were followed for an average of 7 years (4.5 to 10 years), for a total of 2696 person-years; 62 (14.2\%) people developed diabetes during the time they were followed. Individuals scoring 55 points based on their risk factors demonstrated a significantly higher risk of developing diabetes in 7 years ( $R R=6.1$; IC95\%: 1.7 to 11.1); the risk was even higher for those with a score of 75 points ( $R R=9.4$; IC95\%: 2.1 to 11.5 ).

Conclusions. The ITD is easy to use and a valid screening alternative for type 2 diabetes. Its use will allow more individuals to benefit from disease prevention methods and early diagnosis without substantially increasing costs and with minimal use of laboratory resources.

Key words Diabetes mellitus, type 2; risk factors; mass screening; monitoring; Mexico. 